\title{
PHYSICS AND MATHEMATICS
}

\section{THE METHOD OF SUCCESSIVE APPROXIMATIONS IN THE MATHEMATICAL THE-ORY OF SHALLOW SHELLS OF ARBITRARY THICKNESS}

\author{
Candidate of Physical and Mathematical Sciences, Zelensky A. G., \\ Ukraine, Dnipro, Associate Professor of the Department of Building Mechanics and Materials \\ Resistance of the State Higher Educational Institution \\ "Pridneprovsk State Academy of Civil Engineering and Architecture"
}

DOI: https://doi.org/10.31435/rsglobal_ws/30112019/6764

\section{ARTICLE INFO}

Received: 20 September 2019

Accepted: 11 November 2019

Published: 30 November 2019

\section{KEYWORDS}

mathematical theory of transversalisotropic shallow shells of arbitrary thickness, Legendre poly-nomials, method of successive approximations.

\begin{abstract}
The method of sequential approximations (MSA) in mathematical theory (MT) of transversal-isotropic shallow shells of arbitrary thickness is developed. MT takes into account all components of stress-strain state (SSS). SSS and boundary conditions are considered to be functions of three varia-bles. Three-dimensional problems are reduced to twodimensional decompositions of all the compo-nents of the SSS into series in the transverse coordinate using Legendre polynomials and using the Reisner variational principle. The boundary conditions for stresses on the front surfaces of the shell are fulfilled precisely. Previous studies have shown the high efficiency of this MT. The boundary-value problem for a shallow shell is reduced to sequences of two boundary-value problems for the respective plates. One sequence describes symmetric deformation relative to the median plane, and the other sequence is skew symmetric. MSA makes it easier to find a common solution of differential equations (DE) for shallow shells. Highly accurate results for SSS are already in the first approxi-mation. MSA can be used when solving problems for shallow shells by other theories.
\end{abstract}

Citation: Zelensky A. G. (2019) The Method of Successive Approximations in the Mathematical The-Ory
of Shallow Shells of Arbitrary Thickness. World Science. 11(51), Vol.1. doi: 10.31435/rsglobal_ws/30112019/6764

Copyright: (C) 2019 Zelensky A. G. This is an open-access article distributed under the terms of the Creative Commons Attribution License (CC BY). The use, distribution or reproduction in other forums is permitted, provided the original author(s) or licensor are credited and that the original publication in this journal is cited, in accordance with accepted academic practice. No use, distribution or reproduction is permitted which does not comply with these terms.

\section{Introduction.}

Problem solving for shells and plates is performed on the basis of classical and refining theories, using equations of three-dimensional elastic theory and on the basis of variants of mathematical theory. Classical and clarifying theories are based on various physico-geometric assumptions $[1-3,8,12,15,17$, $20,22,23]$. The limits of using these theories for different classes of problems require further research. The most common in practical use are the theories of the Tymoshenko-Reisner type $[20,22,23]$ and their various modifications $[3,8,12,17]$. Clarifying theories include theories based on specific deformation models [11]. The main drawback of all the clarifying theories is the inability to increase the accuracy of the solution of the problems within these theories.

The use of three-dimensional elasticity theory in the analytical solution of boundary value problems for plates and shells $[6,14]$ is too much of a problem for mathematical physics, since all the components of the SSS and boundary conditions are functions of three coordinates. At the same time, three-dimensional SSS occurs in thick plates and shells, in the field of local, discontinuous and nonsmooth loads, under the action of other SSS concentrators. And so there is an urgent need to develop 
and construct theories that take into account all the components of the SSS and boundary effects as a functions of the three variables. And so that these theories can be used to analytically solve boundary value problems. with the required accuracy. These qualities are satisfied by the variants of MT, which are based on a mathematical approach in the image of the components of the SSS with infinite rows in transverse coordinates. These theories are devoid of physico-geometric assumptions. Different mathematical series are used: tensor [9], power [13], using the Lezhran-dra polynomials [4, 5, 7, 10, 16, 18, 19, 24]. Three-dimensional problems are reduced to two-dimensional by different methods: operating $[4,5,24]$, variational $[7,10,16,18,19]$, others [15]. The MT variants have different accuracy depending on the approach of reducing three-dimensional problems to two-dimensional ones and the method of representing the SSS in the form of mathematical series.

In this article, MSA is developed in solving boundary value problems for transversely isotropic shallow shells of arbitrary thickness based on the MT variant [25-28]. Shells can be subjected to arbitrary transverse loads. All SSS components that are functions of three variables are taken into account. The MT is based on the representation of the SSS components in the form of infinite rows with a transverse coordinate using Legendre polynomials. The transverse normal and tangent stresses are approximated by taking into account the three-dimensional DE equilibrium theory of elasticity such that the boundary conditions in the stresses on the face surfaces are satisfied exactly. Three-dimensional problems for shells are reduced to two-dimensional problems based on the Reisner variational principle [21]. This method of constructing the MT variant showed efficiency and high accuracy $[25,26]$. As the number of additives in the mathematical series increases, the order of the systems of equations and the complexity of solving them increases, but the accuracy of the solution increases. The MSA makes it possible to reduce the complex boundary-value problem for the shell to simpler boundary-value problems for the corresponding plates with symmetric and oblique deformation relative to the median plane.

\section{Problem statement.}

We study the transversal isotropic shallow shell of constant arbitrary thickness $h$ h in a rectangular coordinate system $x, y, z$. The surface of the isotropy coincides with the median surface. The axes $x, y$ belong to the plan of the shell, and the axis $z$ is perpendicular to the plane of the plan of the shell and is directed in the direction of the convexity (up) $(-h / 2 \leq z \leq h / 2)$. On the upper and lower surfaces of the shell there is a static transverse load $q_{1}(x, y)$ and $q_{2}(x, y)$ directed downwards. All SSS components are functions of three coordinates. Boundary conditions on the front surfaces:

$$
\sigma_{z}(z=h / 2)=-q_{1}(x, y) ; \sigma_{z}(z=-h / 2)=q_{2}(x, y) ; \sigma_{x z}(z= \pm h / 2)=\sigma_{y z}(z= \pm h / 2)=0
$$

The transverse loads on the upper and lower surfaces are depicted as the sum of two additions: oblique symmetric $q / 2$ and symmetric $p / 2$ loads relative to the middle surface:

$$
\sigma_{z}(z= \pm h / 2)=(\mp q(x, y)-p(x, y)) / 2, p(x, y)=q_{1}(x, y)-q_{2}(x, y), q(x, y)=q_{1}(x, y)+q_{2}(x, y) .
$$

The boundary conditions on the side surface may be different.

The displacement components are represented by the Fourier-Legendre series in the coordinate $z$ :

$$
U(x, y, z)=\sum_{k=0}^{\infty} P_{k}(2 z / h) u_{k}(x, y),\left(U, V ; u_{k}, v_{k}\right) ; W(x, y, z)=\sum_{k=1}^{\infty} P_{k-1}(2 z / h) w_{k}(x, y),
$$

where $P_{k}(2 z / h)$ is Legendre polynomials; $u_{k}, v_{k}, w_{k}$ - sought components in displacements.

If in (2) in tangential displacements we take into account terms with indices $k=0,1,2, \ldots, n$, then we call this approximation K0-n. If we take into account additives with $k=0,1,2,3$ indexes, this is an approximation of $\mathrm{K} 0123$ or $\mathrm{K} 0-3$.

Since the shell is of arbitrary thickness, tangential displacements are taken into account in the shear deformations of $\gamma_{x z}, \gamma_{y z}$ [1] (in the theory of thin shells they are neglected):

$$
\begin{gathered}
\varepsilon_{x}=\partial U / \partial x+k_{1} W ; \varepsilon_{y}=\partial V / \partial y+k_{2} W ; \varepsilon_{z}=\partial W / \partial z ; \gamma_{x y}=\partial U / \partial y+\partial V / \partial x ; \\
\gamma_{x z}=\partial W / \partial x+\partial U / \partial z-k_{1}^{\prime} U,\left(x, y ; U \rightarrow V ; k_{1}^{\prime} \rightarrow k_{2}^{\prime}\right),\left(k_{i}=1 / R_{i} ; k_{i}^{\prime}=k_{i}, i=1,2\right),
\end{gathered}
$$

where $R_{1}, R_{2}$ is the principal radii of curvature of the middle surface of the shell. Clarifying additives in the expressions for the transverse angular deformations contain $k_{1}^{\prime}, k_{2}^{\prime}$.

Here are general structural formulas for stress components [25], which derive from the DE system of the spatial theory of elasticity and the Reisner variational equation: 


$$
\begin{aligned}
& \sigma_{x z}(x, y, z)=\sum_{i=0}^{\infty} P_{i} t_{x i} ; \sigma_{y z}(x, y, z)=\sum_{i=0}^{\infty} P_{i} t_{y i} ; \sigma_{z}(x, y, z)=\sum_{i=0}^{\infty} P_{i} s_{z i} ; \\
& \sigma_{x}(x, y, z)=\sum_{i=0}^{\infty} P_{i} s_{x i},\left(\sigma_{x} \rightarrow \sigma_{y} ; s_{x i} \rightarrow s_{y i}\right) ; \sigma_{y x}(x, y, z)=\sum_{i=0}^{\infty} P_{i} t_{y x i},
\end{aligned}
$$

where $t_{x i}, \ldots, t_{y x i}$-functions that depend on the displacements of $u_{k}(x, y), v_{k}(x, y), w_{k}(x, y)$ and mechanical-geometric parameters (MGP).

3. Displacements, stresses and boundary conditions in the K0-n approximation

3.1. Components of displacements and stresses in the shell. The displacement components are determined according to (2):

$$
U(x, y, z)=\sum_{k=0}^{n} P_{k}(2 z / h) u_{k}(x, y),\left(U, V ; u_{k}, v_{k}\right) ; W(x, y, z)=\sum_{k=1}^{n} P_{k-1}(2 z / h) w_{k}(x, y),
$$

The stress components according to (3):

$$
\begin{aligned}
& \sigma_{x z}(x, y, z)=\sum_{i=0}^{n+1} P_{i} t_{x i} ; \sigma_{y z}(x, y, z)=\sum_{i=0}^{n+1} P_{i} t_{y i} ; \sigma_{z}(x, y, z)=\sum_{i=0}^{n+2} P_{i} s_{z i} \\
& \sigma_{x}(x, y, z)=\sum_{i=0}^{n+2} P_{i} s_{x i} ; \sigma_{y}(x, y, z)=\sum_{i=0}^{n+2} P_{i} s_{y i} ; \sigma_{x y}(x, y, z)=\sum_{i=0}^{n} P_{i} t_{y x i} .
\end{aligned}
$$

The transverse normal and tangent stresses satisfy exactly the conditions (1).

For the approximations K0-3 and K0-5, the functions are given in [26].

3.2. Boundary conditions. The boundary conditions are obtained from the Reisner variation equation:

$$
\begin{gathered}
\int_{(s)}\left\{\sum_{j=0}^{n} \frac{h}{(2 j+1)}\left(\left(s_{x j} l_{x}+t_{y x j} l_{y}-x_{s j}\right) \delta u_{j}+\left(t_{y x j} l_{x}+s_{y j} l_{y}-y_{s j}\right) \delta v_{j}\right)+\right. \\
\left.\left.+\sum_{j=0}^{n-1} \frac{h}{(2 j+1)}\left(t_{x j} l_{x}+t_{y j} l_{y}-z_{s j}\right) \delta w_{j+1}\right)\right\} d s=0,
\end{gathered}
$$

In (6) $l_{x}, l_{y}-$ is the cosines of the angles between the normal vector to the lateral surface and the coordinate axes; $S$ - contour of the shell; $x_{s j}(x, y), y_{s j}(x, y), z_{s j}(x, y)$ - members in mathematical series of the image of the external loading $X_{v}(x, y, z), Y_{v}(x, y, z), Z_{v}(x, y, z)$ by Legendre polynomials:

$$
\begin{gathered}
x_{s j}(x, y)=(2 j+1)\left(\int_{-h / 2}^{h / 2} X_{v}(x, y, z) P_{j}(2 z / h) d z\right) / h,\left(x_{s j} \rightarrow y_{s j} ; X_{v} \rightarrow Y_{v} ; j=0,1, \ldots, n\right) ; \\
z_{s j}(x, y)=(2 j+1)\left(\int_{-h / 2}^{h / 2} X_{v}(x, y, z) P_{j}(2 z / h) d z\right) / h,(j=0,1, \ldots, n-1),
\end{gathered}
$$

where $Z_{n}$ must balance the transverse load on the upper and lower surfaces of the shell.

Equations (6) and (7) yield different boundary conditions. Here are some of them.

1) Boundary conditions in displacements. Only the displacement components $U_{\Gamma}(x, y, z)$, $V_{\Gamma}(x, y, z), W_{\Gamma}(x, y, z)$ are known on the side surface $\Gamma$ of the shell. Boundary conditions:

$$
\begin{gathered}
u_{j}(x, y)=u_{j \Gamma}(x, y) ; v_{j}(x, y)=v_{j \Gamma}(x, y),(j=0,1, \ldots, n) \\
w_{j}(x, y)=w_{j \Gamma}(x, y),(j=1, \ldots, n) ; x, y \in S
\end{gathered}
$$

where

$$
\begin{gathered}
\left.u_{j \Gamma}(x, y)=\frac{2 j+1}{h} \int_{z} U_{\Gamma}(x, y, z) P_{j}(2 z / h) d z,\left(u_{j \Gamma} \Rightarrow v_{j \Gamma_{2}}, U_{\Gamma} \rightarrow V_{\Gamma}\right),(j=0,1, \ldots, n)\right) ; \\
w_{j \Gamma}(x, y)=\frac{2 j-1}{h} \int_{z} W_{\Gamma}(x, y, z) P_{j-1}(2 z / h) d z,(j=1, \ldots, n) .
\end{gathered}
$$

2) Boundary conditions in stresses. Only the external load $X_{v}(x, y, z), \quad Y_{v}(x, y, z)$, $Z_{v}(x, y, z)$ is specified on the side surface. Then we have the following boundary conditions: 


$$
\begin{aligned}
& s_{x j}(x, y) l_{x}+t_{y x j}(x, y) l_{y}=x_{s j}(x, y) ; t_{y x j}(x, y) l_{x}+s_{y j}(x, y) l_{y}=y_{s j}(x, y), \\
& (j=0,1, \ldots, n) ; t_{x j}(x, y) l_{x}+t_{y j}(x, y) l_{y}=z_{s j}(x, y),(j=0,1, \ldots, n-1) ; x, y \in S .
\end{aligned}
$$

3) The boundary conditions for the freely fixed at the edges of the shells:

$v_{j}(x=0, y)=v_{j}(x=a, y)=0,(j=0,1, \ldots, n) ; w_{j}(x=0, y)=w_{j}(x=a, y)=0,(j=1, \ldots, n)$;

$$
s_{x j}(x=0, y)=s_{x j}(x=a, y)=0,(j=0,1, \ldots, n) ;
$$

$u_{j}(x, y=0)=u_{j}(x, y=b)=0,(j=0,1, \ldots, n) ; w_{j}(x, y=0)=w_{j}(x, y=b)=0,(j=1, \ldots, n)$;

$$
s_{y j}(x, y=0)=s_{y j}(x, y=b)=0,(j=0,1, \ldots, n) .
$$

\section{4) Boundary conditions for rigidly secured shells:}

$$
\begin{gathered}
u_{j}(x=0, y)=u_{j}(x=a, y)=0, v_{j}(x, y=0)=v_{j}(x, y=b)=0,(j=0,1, \ldots, n) ; \\
w_{j}(x=0, y)=w_{j}(x=a, y)=w_{j}(x, y=0)=w_{j}(x, y=b)=0,(j=1, \ldots, n) .
\end{gathered}
$$

In the approximations $\mathrm{K} 01, \mathrm{~K} 0-3, \mathrm{~K} 0-5$, to obtain displacements, stresses and boundary conditions, it is necessary to put $n=1 ; n=3 ; n=5$ in (4) - (11), respectively.

\section{The method of successive approximations}

4.1. The K0-3 approximation. The system of equilibrium DE has the 22nd order:

$$
\begin{gathered}
D_{j, 1} u_{0}+D_{j, 2} v_{0}+D_{j, 3} u_{1}+D_{j, 4} v_{1}+D_{j, 5} u_{2}+D_{j, 6} v_{2}+D_{j, 7} u_{3}+ \\
+D_{j, 8} v_{3}+D_{j, 9} w_{1}+D_{j, 10} w_{2}+D_{j, 11} w_{3}=D_{j p q}(x, y),(j=1,2, \ldots, 11),
\end{gathered}
$$

where

$$
\begin{aligned}
& D_{1,1}=\gamma_{111} \frac{\partial^{2}}{\partial x^{2}}+\gamma_{112} \frac{\partial^{2}}{\partial y^{2}}+k_{1}^{\prime} l_{1 x 0}, \quad D_{1,2}=\gamma_{121} \frac{\partial^{2}}{\partial x \partial y}, D_{1,3}=k_{1}^{\prime} l_{1 x 1}, D_{1,4}=0, \\
& D_{1,5}=\gamma_{131} \frac{\partial^{2}}{\partial x^{2}}+k_{1}^{\prime} l_{1 x 2}, \quad D_{1,6}=\gamma_{131} \frac{\partial^{2}}{\partial x \partial y}, \quad D_{1,7}=k_{1}^{\prime} l_{1 x 3}, \quad D_{1,8}=0, D_{1,9}=k_{1 w 1} \frac{\partial}{\partial x}, D_{1,10}=\gamma_{151} \frac{\partial}{\partial x}, \\
& D_{1,11}=k_{1 w 3} \frac{\partial}{\partial x}, D_{1 p q}=\gamma_{u 0} \frac{\partial p}{\partial x}, D_{2,2}=\gamma_{112} \frac{\partial^{2}}{\partial x^{2}}+\gamma_{111} \frac{\partial^{2}}{\partial y^{2}}+k_{2}^{\prime} l_{1 y 0}, D_{2,3}=0, D_{2,4}=k_{2}^{\prime} l_{1 y 1}, \\
& D_{2,5}=\gamma_{131} \frac{\partial^{2}}{\partial x \partial y}, D_{2,6}=\gamma_{131} \frac{\partial^{2}}{\partial y^{2}}+k_{2}^{\prime} l_{1 y 2}, \quad D_{2,7}=0, D_{2,8}=k_{2}^{\prime} l_{1 y 3}, D_{2,9}=k_{2 w 1} \frac{\partial}{\partial y}, \\
& D_{2,10}=\gamma_{151} \frac{\partial}{\partial y}, D_{2,11}=k_{2 w 3} \frac{\partial}{\partial y}, D_{2 p q}=\gamma_{u 0} \frac{\partial p}{\partial y} ; D_{3,3}=\beta_{111} \frac{\partial^{2}}{\partial x^{2}}+\beta_{112} \frac{\partial^{2}}{\partial y^{2}}+\beta_{113}+k_{1}^{\prime} l_{2 x 1} / 5, \\
& D_{3,4}=\beta_{121} \frac{\partial^{2}}{\partial x \partial y}, D_{3,5}=k_{1}^{\prime} k_{3 u 2}, D_{3,6}=0, D_{3,7}=\beta_{131} \frac{\partial^{2}}{\partial x^{2}}+\beta_{133}+k_{1}^{\prime} l_{2 x 3} / 5, D_{3,8}=\beta_{131} \frac{\partial^{2}}{\partial x \partial y}, \\
& D_{3,9}=\beta_{151} \frac{\partial}{\partial x}, D_{3,10}=k_{3 w 2} \frac{\partial}{\partial x}, D_{3,11}=\beta_{161} \frac{\partial}{\partial x}, D_{3 p q}=\beta_{u 1} \frac{\partial q}{\partial x}, \\
& D_{4,4}=\beta_{112} \frac{\partial^{2}}{\partial x^{2}}+\beta_{111} \frac{\partial^{2}}{\partial y^{2}}+\beta_{113}+k_{2}^{\prime} l_{2 y 1} / 5, D_{4,5}=0, D_{4,6}=k_{2}^{\prime} k_{3 u 2}, D_{4,7}=\beta_{131} \frac{\partial^{2}}{\partial x \partial y}, \\
& D_{4,8}=\beta_{131} \frac{\partial^{2}}{\partial y^{2}}+\beta_{133}+k_{2}^{\prime} l_{2 y 3} / 5, D_{4,9}=\beta_{151} \frac{\partial}{\partial y}, D_{4,10}=k_{4 w 2} \frac{\partial}{\partial y}, D_{4,11}=\beta_{161} \frac{\partial}{\partial y}, D_{4 p q}=\beta_{u 1} \frac{\partial q}{\partial y} ; \\
& D_{5,5}=\gamma_{331} \frac{\partial^{2}}{\partial x^{2}}+\gamma_{332} \frac{\partial^{2}}{\partial y^{2}}+\gamma_{333}+\left(k_{1}^{\prime}\right)^{2} k_{5 u 2}, D_{5,6}=\gamma_{341} \frac{\partial^{2}}{\partial x \partial y}, D_{5,7}=k_{1}^{\prime} k_{5 u 3}, D_{5,8}=0 \text {, } \\
& D_{5,9}=k_{5 w 1} \frac{\partial}{\partial x}, D_{5,10}=\gamma_{351} \frac{\partial}{\partial x}, D_{5,11}=k_{5 w 3} \frac{\partial}{\partial x}, D_{5 p q}=\gamma_{u 2} \frac{\partial p}{\partial x}, \\
& D_{6,6}=\gamma_{332} \frac{\partial^{2}}{\partial x^{2}}+\gamma_{331} \frac{\partial^{2}}{\partial y^{2}}+\gamma_{333}+\left(k_{2}^{\prime}\right)^{2} k_{6 v 2}, D_{6,7}=0, D_{6,8}=k_{2}^{\prime} k_{5 u 3}, D_{6,9}=k_{6 w 1} \frac{\partial}{\partial y} \quad \text {, }
\end{aligned}
$$




$$
\begin{gathered}
D_{6,10}=\gamma_{351} \frac{\partial}{\partial y}, D_{6,11}=k_{6 w 3} \frac{\partial}{\partial y}, D_{6 p q}=\gamma_{u 2} \frac{\partial p}{\partial y}, D_{7,7}=\beta_{331} \frac{\partial^{2}}{\partial x^{2}}+\beta_{332} \frac{\partial^{2}}{\partial y^{2}}+\beta_{333}+\left(k_{1}^{\prime}\right)^{2} k_{7 u 3}, \\
D_{7,8}=\beta_{341} \frac{\partial}{\partial x \partial y}, D_{7,9}=\beta_{351} \frac{\partial}{\partial x}, D_{7,10}=k_{7 w 2} \frac{\partial}{\partial x}, D_{7,11}=\beta_{361} \frac{\partial}{\partial x}, D_{7 p q}=\beta_{u 3} \frac{\partial q}{\partial x} \\
D_{8,8}=\beta_{332} \frac{\partial^{2}}{\partial x^{2}}+\beta_{331} \frac{\partial^{2}}{\partial y^{2}}+\beta_{333}+\left(k_{2}^{\prime}\right)^{2} k_{8 v 3}, D_{8,9}=\beta_{351} \frac{\partial}{\partial y}, D_{8,10}=k_{8 w 2} \frac{\partial}{\partial y}, D_{8,11}=\beta_{361} \frac{\partial}{\partial y}, \\
D_{8 p q}=\beta_{u 3} \frac{\partial q}{\partial y}, D_{9,9}=\beta_{551} \nabla^{2}+r_{1 w 1}, D_{9,10}=r_{1 w 2}, D_{9,11}=\beta_{561} \nabla^{2}+r_{1 w 3}, D_{9 p q}=k_{9 p} p+\beta_{w 1} q, \\
D_{10,10}=\gamma_{551} \nabla^{2}+\gamma_{552}+r_{2 w 2}, D_{10,11}=r_{2 w 3}, D_{10 p q}=k_{10 q} q+\gamma_{w 2} p, D_{11,11}=\beta_{661} \nabla^{2}+\beta_{662}+r_{3 w 3}, \\
D_{11 p q}=\beta_{w 3} q+k_{11 p} p .
\end{gathered}
$$

It is shown that the differential matrix of the DE (12) system is symmetric $\left(D_{i j}=D_{j i}\right)$. In (13):

$$
\begin{gathered}
\gamma_{111}=h\left(d_{0}-\frac{1}{10} d_{10} e_{20}\right), \gamma_{112}=h G, \gamma_{121}=h\left(G+d_{0} v-\frac{1}{10} d_{10} e_{20}\right), \gamma_{131}=-\frac{h}{10} d_{10} e_{22}, \\
\gamma_{131}=-\frac{h}{10} d_{10} e_{22}, k_{1 w 1}=\left(d_{0} k_{1 v}-\frac{k_{12}}{10} d_{10} e_{20}+\frac{k_{1}^{\prime}}{h} h_{11}\right) h, \gamma_{151}=-\frac{h}{10} d_{10} q_{22}, \\
k_{1 w 3}=\left(\frac{k_{1}^{\prime}}{h} h_{13}-\frac{k_{12}}{10} d_{10} e_{22}\right) h, \gamma_{u 0}=\frac{3 h}{20} d_{10} ; k_{2 w 1}=\left(d_{0} k_{2 v}-\frac{k_{12}}{10} d_{10} e_{20}+\frac{k_{2}^{\prime}}{h} h_{11}\right) h, \\
k_{2 w 3}=\left(\frac{k_{2}^{\prime}}{h} h_{13}-\frac{k_{12}}{10} d_{10} e_{22}\right) h ; \beta_{111}=\frac{h}{3}\left(d_{0}-\frac{3}{70} d_{10} e_{31}\right), \beta_{112}=\frac{1}{3} h G, \beta_{113}=-\frac{2}{h} l_{1 x 1}, \\
\beta_{121}=\frac{h}{3}\left(G+d_{0} v-\frac{3}{70} d_{10} e_{31}\right), k_{3 u 2}=\frac{19}{15} G^{\prime}, \beta_{131}=-\frac{h}{70} d_{10} e_{33}, \beta_{133}=-\frac{2}{h} l_{1 x 3}, \beta_{151}=-\frac{2}{h} h_{11},
\end{gathered}
$$$$
k_{3 w 2}=\frac{h}{3} d_{0} k_{1 v}+\frac{k_{1}^{\prime}}{5} h_{22}-\frac{1}{70} h d_{10} q_{32}, \beta_{161}=-\frac{2}{h} h_{13}-\frac{h}{70} d_{10} q_{33}, \beta_{u 1}=\frac{2}{21} h d_{10} ;
$$$$
k_{4 w 2}=\frac{h}{3} d_{0} k_{2 v}+\frac{k_{2}^{\prime}}{5} h_{22}-\frac{1}{70} h d_{10} q_{32}, \gamma_{331}=\frac{h}{5}\left(d_{0}+\frac{1}{7} d_{10} e_{22}\right), \gamma_{332}=\frac{1}{5} h G, \gamma_{333}=-\frac{6}{5 h} l_{2 \times 2} \text {, }
$$$$
k_{5 u 2}=k_{6 v 2}=-\frac{2}{15} G^{\prime} h, \gamma_{341}=\frac{h}{5}\left(G+d_{0} v+\frac{1}{7} d_{10} e_{22}\right), k_{5 u 3}=\frac{3}{5} G^{\prime}, k_{5 w 1}=\frac{h}{35} k_{12} d_{10} e_{20}-\frac{k_{1}^{\prime}}{15} G^{\prime} h \text {, }
$$$$
\gamma_{351}=\frac{1}{5}\left(\frac{h}{7} d_{10} q_{22}-\frac{6}{h} h_{22}\right), k_{5 w 3}=\frac{k_{12}}{35} h d_{10} e_{22}+\frac{h}{5} k_{1 v} d_{0}+\frac{2}{15} k_{1}^{\prime} h G^{\prime}, \gamma_{u 2}=\frac{1}{10} h d_{10} ;
$$$$
k_{6 w 1}=\frac{h}{35} k_{12} d_{10} e_{20}-\frac{k_{2}^{\prime}}{15} h G^{\prime}, k_{6 w 3}=\frac{1}{5}\left(\frac{k_{12}}{7} h d_{10} e_{22}+k_{2 v} h d_{0}+\frac{2}{3} k_{2}^{\prime} h G^{\prime}\right),
$$$$
\beta_{331}=\frac{h}{7}\left(d_{0}+\frac{1}{15} d_{10} e_{33}\right), \beta_{332}=\frac{1}{7} h G, \beta_{333}=-\frac{6}{7 h} l_{3 \times 3}, k_{7 u 3}=k_{8 v 3}=-\frac{3}{70} G^{\prime} h,
$$$$
\beta_{341}=\frac{h}{7}\left(G+d_{0} v+\frac{1}{15} d_{10} e_{33}\right), \beta_{351}=-\frac{6}{7 h} h_{31}, k_{7 w 2}=\frac{1}{35}\left(\frac{1}{3} h d_{10} q_{32}-3 k_{1}^{\prime} h_{22}\right),
$$$$
\beta_{361}=\frac{1}{7}\left(\frac{1}{15} h d_{10} q_{33}-\frac{6}{h} h_{33}\right), k_{8 w 2}=\frac{1}{35}\left(\frac{1}{3} h d_{10} q_{32}-3 k_{2}^{\prime} h_{22}\right), \beta_{u 3}=\frac{h}{18} d_{10}, \beta_{551}=-h_{11} \text {, }
$$

$r_{1 w 1}=h\left(k_{v} d_{0}-\frac{k_{12}^{2}}{10} d_{10} e_{20}\right), r_{1 w 2}=-\frac{k_{12}}{10} h d_{10} q_{22}, \beta_{551}=-h_{11}, \beta_{561}=-h_{13}, r_{1 w 3}=-\frac{k_{12}^{2}}{10} h d_{10} e_{22}$,

$$
\begin{gathered}
k_{9 p}=\frac{3 k_{12}}{20} h d_{10}, \beta_{w 1}=-1, \gamma_{551}=-\frac{1}{5} h_{22}, \gamma_{552}=-\frac{1}{5} q_{22}, r_{2 w 2}=h\left(\frac{1}{3} k_{v} d_{0}-\frac{k_{12}}{70} d_{10} q_{32}\right), \\
r_{2 w 3}=-\frac{k_{12}}{5}\left(e_{22}+\frac{1}{14} h d_{10} q_{33}\right), k_{10 q}=\frac{2 k_{12}}{21} h d_{10}, \gamma_{w 2}=-\frac{7}{10}, \beta_{661}=-\frac{2}{15} h G^{\prime}, \beta_{662}=-\frac{3}{35} q_{33},
\end{gathered}
$$




$$
\begin{gathered}
r_{3 w 3}=\frac{h}{5}\left(k_{v} d_{0}+\frac{k_{12}^{2}}{7} d_{10} e_{22}\right), \beta_{w 3}=-\frac{3}{7}, k_{11 p}=\frac{k_{12}}{10} h d_{10} ; h_{11}=\frac{14}{15} G^{\prime} h, h_{13}=-\frac{1}{15} G^{\prime} h, h_{22}=\frac{7}{6} G^{\prime} h, \\
h_{31}=\frac{7}{5} G^{\prime} h, h_{33}=h_{11},\left(x \rightarrow y ; k_{1}^{\prime} \rightarrow k_{2}^{\prime}\right) ; q_{21}=-7 d_{30} k_{12}, q_{22}=-\frac{14}{h d_{20}}, q_{23}=2 d_{30} k_{12}, \\
q_{32}=-11 d_{30} k_{12}, q_{33}=-\frac{66}{h d_{20}}, e_{20}=-7 d_{30} ; e_{22}=2 d_{30} ; e_{31}=-11 d_{30} ; e_{33}=\frac{22}{3} d_{30} ; e_{2 p}=-\frac{7}{2} ; \\
e_{3 q}=-\frac{22}{3} ; d_{0}=E /\left(1-v^{2}\right), d_{10}=E v^{\prime} /\left(E^{\prime}(1-v)\right), d_{20}=\left(1-2 d_{10} v^{\prime}\right) / E^{\prime}, d_{30}=d_{10} / d_{20} ; \\
k_{1 v}=k_{1}+k_{2} v, k_{2 v}=k_{2}+k_{1} v, k_{v}=k_{1} k_{1 v}+k_{2} k_{2 v},
\end{gathered}
$$

where $E, E^{\prime}, v, v^{\prime}, G, G^{\prime}$ is the mechanical parameters of the transversely isotropic material.

The DE system (12) is not divided into two systems that describe independently symmetric and oblique deformation. This indicates the interdependence of symmetric and oblique deformation of the shells. For plates, DE systems are separated.

To obtain the MSA equations, we transfer all the additions of the left-hand sides of equations (12) containing the curvatures of the shell to the right-hand side. We will have the following system in the $i=1,2, \ldots$ approximation:

$$
\begin{aligned}
& L_{j, 1} u_{0}^{(i)}+L_{j, 2} v_{0}^{(i)}+L_{j, 3} u_{1}^{(i)}+L_{j, 4} v_{1}^{(i)}+L_{j, 5} u_{2}^{(i)}+L_{j, 6} v_{2}^{(i)}+L_{j, 7} u_{3}^{(i)}+ \\
+ & L_{j, 8} v_{3}^{(i)}+L_{j, 9} w_{1}^{(i)}+L_{j, 10} w_{2}^{(i)}+L_{j, 11} w_{3}^{(i)}=L_{i p q}^{(i-1)}(x, y),(j=1,2, \ldots, 11),
\end{aligned}
$$

where

$$
\begin{aligned}
& L_{1,1}=\gamma_{111} \frac{\partial^{2}}{\partial x^{2}}+\gamma_{112} \frac{\partial^{2}}{\partial y^{2}}, L_{1,2}=\gamma_{121} \frac{\partial^{2}}{\partial x \partial y}, L_{1,3}=L_{1,4}=0 \\
& L_{1,5}=\gamma_{131} \frac{\partial^{2}}{\partial x^{2}}, L_{1,6}=\gamma_{131} \frac{\partial^{2}}{\partial x \partial y}, L_{1,7}=L_{1,8}=L_{1,9}=0, L_{1,10}=\gamma_{151} \frac{\partial}{\partial x}, L_{1,11}=0 \text {, } \\
& L_{1 p q}^{(i-1)}=\gamma_{u 0} p_{, x}-\left(k_{1}^{\prime} l_{1 x 0} u_{0}+k_{1}^{\prime} l_{1 \times 1} u_{1}+k_{1}^{\prime} l_{1 \times 2} u_{2}+k_{1}^{\prime} l_{1 \times 3} u_{3}+k_{1 w 1} w_{1, x}+k_{1 w 3} w_{3, x}\right)^{(i-1)} \text {; } \\
& L_{2,2}=\gamma_{112} \frac{\partial^{2}}{\partial x^{2}}+\gamma_{111} \frac{\partial^{2}}{\partial y^{2}}, L_{2,3}=L_{2,4}=0, L_{2,5}=\gamma_{131} \frac{\partial^{2}}{\partial x \partial y}, L_{2,6}=\gamma_{131} \frac{\partial^{2}}{\partial y^{2}}, \\
& L_{2,7}=L_{2,8}=L_{2,9}=0, L_{2,10}=\gamma_{151} \frac{\partial}{\partial y}, L_{2,11}=0, \\
& L_{2 p q}^{(i-1)}=\gamma_{u 0} p_{, y}-\left(k_{2}^{\prime} l_{1 y 0} v_{0}+k_{2}^{\prime} l_{1 y 1} v_{1}+k_{2}^{\prime} l_{1 y 2} v_{2}+k_{2}^{\prime} l_{1 y 3} v_{3}+k_{2 w 1} w_{1, y}+k_{2 w 3} w_{3, y}\right)^{(i-1)} \text {; } \\
& L_{3,3}=\beta_{111} \frac{\partial^{2}}{\partial x^{2}}+\beta_{112} \frac{\partial^{2}}{\partial y^{2}}+\beta_{113}, L_{3,4}=\beta_{121} \frac{\partial^{2}}{\partial x \partial y}, L_{3,5}=L_{3,6}=0, L_{3,7}=\beta_{131} \frac{\partial^{2}}{\partial x^{2}}+\beta_{133} \text {, } \\
& L_{3,8}=\beta_{131} \frac{\partial^{2}}{\partial x \partial y}, L_{3,9}=\beta_{151} \frac{\partial}{\partial x}, L_{3,10}=0, L_{3,11}=\beta_{161} \frac{\partial}{\partial x}, \\
& L_{3 p q}^{(i-1)}=\beta_{u 1} q_{, x}-\left(k_{1}^{\prime} l_{1 x 1} u_{1}+k_{1}^{\prime} l_{2 x 1} u_{1} / 5+k_{1}^{\prime} k_{3 u 2} u_{2}+k_{1}^{\prime} l_{2 x 3} u_{3} / 5+k_{3 w 2} w_{2, x}\right)^{(i-1)} \text {; } \\
& L_{4,4}=\beta_{112} \frac{\partial^{2}}{\partial x^{2}}+\beta_{111} \frac{\partial^{2}}{\partial y^{2}}+\beta_{113}, L_{4,5}=0, L_{4,6}=0, L_{4,7}=\beta_{131} \frac{\partial^{2}}{\partial x \partial y}, L_{4,8}=\beta_{131} \frac{\partial^{2}}{\partial y^{2}}+\beta_{133} \text {, } \\
& L_{4,9}=\beta_{151} \frac{\partial}{\partial y}, L_{4,10}=0, L_{4,11}=\beta_{161} \frac{\partial}{\partial y}, \\
& L_{4 p q}^{(i-1)}=\beta_{u 1} q_{, y}-\left(k_{2}^{\prime} l_{1 y 1} v_{1}+k_{2}^{\prime} l_{2 y 1} v_{1} / 5+k_{2}^{\prime} k_{3 u 2} v_{2}+k_{2}^{\prime} l_{2 y 3} v_{3} / 5+k_{4 w 2} w_{2, y}\right)^{(i-1)} \text {; } \\
& L_{5,5}=\gamma_{331} \frac{\partial^{2}}{\partial x^{2}}+\gamma_{332} \frac{\partial^{2}}{\partial y^{2}}+\gamma_{333}, L_{5,6}=\gamma_{341} \frac{\partial^{2}}{\partial x \partial y}, \\
& L_{5,7}=L_{5,8}=L_{5,9}=0, L_{5,10}=\gamma_{351} \frac{\partial}{\partial x}, L_{5,11}=0,
\end{aligned}
$$




$$
\begin{aligned}
& L_{5 p q}^{(i-1)}=\gamma_{u 2} p_{, x}-\left(k_{1}^{\prime} l_{1 x 2} u_{0}+k_{1}^{\prime} k_{3 u 2} u_{1}+\left(k_{1}^{\prime}\right)^{2} k_{5 u 2} u_{2}+k_{1}^{\prime} k_{5 u 3} u_{3}+k_{5 w 1} w_{1, x}+k_{5 w 3} w_{3, x}\right)^{(i-1)} \text {; } \\
& L_{6,6}=\gamma_{332} \frac{\partial^{2}}{\partial x^{2}}+\gamma_{331} \frac{\partial^{2}}{\partial y^{2}}+\gamma_{333}, L_{6,7}=L_{6,8}=L_{6,9}=0, L_{6,10}=\gamma_{351} \frac{\partial}{\partial y}, L_{6,11}=0 \text {, } \\
& L_{6 p q}^{(i-1)}=\gamma_{u 2} p_{, y}-\left(k_{2}^{\prime} l_{1 y 2} v_{0}+k_{2}^{\prime} k_{3 u 2} v_{1}+\left(k_{2}^{\prime}\right)^{2} k_{5 u 2} v_{2}+k_{2}^{\prime} k_{5 u 3} v_{3}+k_{6 w 1} w_{1, y}+k_{6 w 3} w_{3, y}\right)^{(i-1)} \\
& L_{7,7}=\beta_{331} \frac{\partial^{2}}{\partial x^{2}}+\beta_{332} \frac{\partial^{2}}{\partial y^{2}}+\beta_{333}, L_{7,8}=\beta_{341} \frac{\partial}{\partial x \partial y}, L_{7,9}=\beta_{351} \frac{\partial}{\partial x}, L_{7,10}=0, \\
& L_{7,11}=\beta_{361} \frac{\partial}{\partial x} ; L_{7 p q}^{(i-1)}=\beta_{u 3} q_{, x}-\left(k_{1}^{\prime} l_{1 \times 3} u_{0}+k_{1}^{\prime} l_{2 \times 3} u_{1} / 5+k_{1}^{\prime} k_{5 u 3} u_{2}+\left(k_{1}^{\prime}\right)^{2} k_{7 u 3} u_{3}+k_{7 w 2} w_{2, x}\right)^{(i-1)} \\
& L_{8,8}=\beta_{332} \frac{\partial^{2}}{\partial x^{2}}+\beta_{331} \frac{\partial^{2}}{\partial y^{2}}+\beta_{333}, L_{8,9}=\beta_{351} \frac{\partial}{\partial y}, L_{8,10}=0, L_{8,11}=\beta_{361} \frac{\partial}{\partial y}, \\
& L_{8 p q}^{(i-1)}=\beta_{u 3} q_{, y}-\left(k_{2}^{\prime} l_{1 y 3} v_{0}+k_{2}^{\prime} l_{2 y 3} v_{1} / 5+k_{2}^{\prime} k_{5 u 3} v_{2}+\left(k_{2}^{\prime}\right)^{2} k_{8 v 3} v_{3}+k_{8 v 2} w_{2, y}\right)^{(i-1)} \text {; } \\
& L_{9,9}=\beta_{551} \nabla^{2}, L_{9,10}=0, L_{9,11}=\beta_{561} \nabla^{2}, L_{10,10}=\gamma_{551} \nabla^{2}+\gamma_{553}, L_{10,11}=0, L_{11,11}=\beta_{661} \nabla^{2}+\beta_{663} \text {, } \\
& L_{9 p q}^{(i-1)}=k_{9 p} p+\beta_{w 1} q-\left(k_{1 w 1} u_{0, x}+k_{2 w 1} v_{0, y}+k_{5 w 1} u_{2, x}+k_{6 w 1} v_{2, y}+r_{1 w 1} w_{1}+r_{1 w 2} w_{2}+r_{1 w 3} w_{3}\right)^{(i-1)} \text {; } \\
& L_{10 p q}^{(i-1)}=\gamma_{w 2} p+k_{10 q} q-\left(k_{3 w 2} u_{1, x}+k_{4 w 2} v_{1, y}+k_{7 w 2} u_{3, x}+k_{8 w 2} v_{3, y}+r_{1 w 2} w_{1}+r_{2 w 2} w_{2}+r_{2 w 3} w_{3}\right)^{(i-1)} \text {; } \\
& L_{11 p q}^{(i-1)}=k_{11 p} p+\beta_{w 3} q-\left(k_{1 w 3} u_{0, x}+k_{2 w 3} v_{0, y}+k_{5 w 3} u_{2, x}+k_{6 w 3} v_{2, y}+r_{1 w 3} w_{1}+r_{2 w 3} w_{2}+r_{3 w 3} w_{3}\right)^{(i-1)} \text {. }
\end{aligned}
$$

In the zero approximation ( $i=0)$, the system of equations (12) has the following form:

$$
\begin{aligned}
& L_{j, 1} u_{0}^{(0)}+L_{j, 2} v_{0}^{(0)}+L_{j, 3} u_{1}^{(0)}+L_{j, 4} v_{1}^{(0)}+L_{j, 5} u_{2}^{(0)}+L_{j, 6} v_{2}^{(0)}+L_{j, 7} u_{3}^{(0)}+ \\
+ & L_{j, 8} v_{3}^{(0)}+L_{j, 9} w_{1}^{(0)}+L_{j, 10} w_{2}^{(0)}+L_{j, 11} w_{3}^{(0)}=L_{j p q}(x, y),(j=1,2, \ldots, 11),
\end{aligned}
$$

where

$$
L_{1 p q}=\gamma_{u 0} p_{, x} ; L_{2 p q}=\gamma_{u 0} p_{, y} ; L_{3 p q}=\beta_{u 1} q_{, x} ; L_{4 p q}=\beta_{u 1} q_{, y} ; L_{5 p q}=\gamma_{u 2} p_{, x} ; L_{6 p q}=\gamma_{u 2} p_{, y} ;
$$
$L_{7 p q}=\beta_{u 3} q_{, x} ; L_{8 p q}=\beta_{u 3} q_{, y} ; L_{9 p q}=k_{9 p q} p+\beta_{w 1} q ; L_{10 p q}=\gamma_{w 2} p+k_{10 q} q ; L_{11 p q}=k_{11 p} p+\beta_{w 3} q$.

Equations 1, 2, 5, 6, 10 of systems (14) and (15) (10th order) describe symmetric deformation of the corresponding plates, and 3, 4, 7-9, 11 - skew symmetric deformation (12th order).

4.2. Approximation K013. In approximation K013, the DE (16th order) system consists of the first-fourth, seventh-ninth, and eleventh equations (12). In addition, you need to put $p(x, y)=0$ and consider only the functions of $u_{0}, v_{0}, u_{1}, v_{1}, u_{3}, v_{3}, w_{1}, w_{3}, q$. System (12) for $i=1,2, \ldots$ then looks like:

$$
\begin{gathered}
L_{j, 1} u_{0}^{(i)}+L_{j, 2} v_{0}^{(i)}+L_{j, 3} u_{1}^{(i)}+L_{j, 4} v_{1}^{(i)}+L_{j, 7} u_{3}^{(i)}+ \\
+L_{j, 8} v_{3}^{(i)}+L_{j, 9} w_{1}^{(i)}+L_{j, 11} w_{3}^{(i)}=L_{i p q}^{(i-1)}(x, y),(j=1,2,3,4,7,8,9,11),
\end{gathered}
$$

where

$$
\begin{gathered}
L_{1,1}=\gamma_{111} \frac{\partial^{2}}{\partial x^{2}}+\gamma_{112} \frac{\partial^{2}}{\partial y^{2}}, L_{1,2}=\gamma_{121} \frac{\partial^{2}}{\partial x \partial y}, L_{1,3}=0, L_{1,4}=0, L_{1,7}=0, L_{1,8}=0, L_{1,9}=0 \\
L_{1,11}=0, L_{1 p q}^{(i-1)}=-\left(k_{1}^{\prime} l_{1 x 0} u_{0}+k_{1}^{\prime} l_{1 x 1} u_{1}+k_{1}^{\prime} l_{1 x 3} u_{3}+k_{1 w 1} w_{1, x}+k_{1 w 3} w_{3, x}\right)^{(i-1)} \\
L_{2,2}=\gamma_{112} \frac{\partial^{2}}{\partial x^{2}}+\gamma_{111} \frac{\partial^{2}}{\partial y^{2}}, L_{2,3}=0, L_{2,4}=0, L_{2,7}=0, L_{2,8}=0, L_{2,9}=0, L_{2,11}=0 \\
L_{3,3}=\beta_{111} \frac{\partial^{2}}{\partial x^{2}}+\beta_{112} \frac{\partial^{2}}{\partial y^{2}}+\beta_{113}, L_{3,4}=\beta_{121} \frac{\partial^{2}}{\partial x \partial y}, L_{3,7}=\beta_{131} \frac{\partial^{2}}{\partial x^{2}}+\beta_{133}, L_{3,8}=\beta_{131} \frac{\partial^{2}}{\partial x \partial y}, \\
L_{3,9}=\beta_{151} \frac{\partial}{\partial x}, L_{3,11}=\beta_{161} \frac{\partial}{\partial x}, L_{3 p q}^{(i-1)}=\beta_{u 1} q, x-\left(k_{1}^{\prime} l_{1 x 1} u_{1}+k_{1}^{\prime} l_{2 x 1} u_{1} / 5+k_{1}^{\prime} l_{2 x 3} u_{3} / 5\right)^{(i-1)}
\end{gathered}
$$




$$
\begin{gathered}
L_{4,4}=\beta_{112} \frac{\partial^{2}}{\partial x^{2}}+\beta_{111} \frac{\partial^{2}}{\partial y^{2}}+\beta_{113}, L_{4,7}=\beta_{131} \frac{\partial^{2}}{\partial x \partial y}, L_{4,8}=\beta_{131} \frac{\partial^{2}}{\partial y^{2}}+\beta_{133}, L_{4,9}=\beta_{151} \frac{\partial}{\partial y}, \\
L_{4,11}=\beta_{161} \frac{\partial}{\partial y}, L_{4 p q}^{(i-1)}=\beta_{u 1} q_{, y}-\left(k_{2}^{\prime} l_{1 y 1} v_{1}+k_{2}^{\prime} l_{2 y 1} v_{1} / 5+k_{2}^{\prime} l_{2 y 3} v_{3} / 5\right)^{(i-1)} \\
L_{7,7}=\beta_{331} \frac{\partial^{2}}{\partial x^{2}}+\beta_{332} \frac{\partial^{2}}{\partial y^{2}}+\beta_{333}, L_{7,8}=\beta_{341} \frac{\partial}{\partial x \partial y}, L_{7,9}=\beta_{351} \frac{\partial}{\partial x}, \\
L_{7,11}=\beta_{361} \frac{\partial}{\partial x}, L_{7 p q}^{(i-1)}=\beta_{u 3} q_{, x}-\left(k_{1}^{\prime} l_{1 x 3} u_{0}+k_{1}^{\prime} l_{2 x 3} u_{1} / 5+\left(k_{1}^{\prime}\right)^{2} k_{7 u 3} u_{3}\right)^{(i-1)} \\
L_{8,8}=\beta_{332} \frac{\partial^{2}}{\partial x^{2}}+\beta_{331} \frac{\partial^{2}}{\partial y^{2}}+\beta_{333}, L_{8,9}=\beta_{351} \frac{\partial}{\partial y}, L_{8,11}=\beta_{361} \frac{\partial}{\partial y}, \\
L_{8 p q}^{(i-1)}=\beta_{u 3} q, y-\left(k_{2}^{\prime} l_{1 y 3} v_{0}+k_{2}^{\prime} l_{2 y 3} v_{1} / 5+\left(k_{2}^{\prime}\right)^{2} k_{8 v 3} v_{3}\right)^{(i-1)} ; \\
L_{9,9}=\beta_{551} \nabla^{2}, L_{9,11}=\beta_{561} \nabla^{2}, L_{9 p q}^{(i-1)}=\beta_{w 1} q-\left(k_{1 w 1} u_{0, x}+k_{2 w 1} v_{0, y}+r_{1 w 1} w_{1}+r_{1 w 3} w_{3}\right)^{(i-1)} \\
L_{11,11}=\beta_{661} \nabla^{2}+\beta_{663}, L_{11 p q}^{(i-1)}=\beta_{w 3} q-\left(k_{1 w 3} u_{0, x}+k_{2 w 3} v_{0, y}+r_{1 w 3} w_{1}+r_{3 w 3} w_{3}\right)^{(i-1)} .
\end{gathered}
$$

In the zero approximation $(i=0)$ the system of $\mathrm{DE}(j=1,2,3,4,7,8,9,11)$ is as follows:

$$
L_{j, 1} u_{0}^{(0)}+L_{j, 2} v_{0}^{(0)}+L_{j, 3} u_{1}^{(0)}+L_{j, 4} v_{1}^{(0)}+L_{j, 7} u_{3}^{(0)}+L_{j, 8} v_{3}^{(0)}+L_{j, 9} w_{1}^{(0)}+L_{j, 11} w_{3}^{(0)}=L_{j p q}(x, y),
$$

where $L_{1 p q}=L_{2 p q} 0 ; L_{3 p q}=\beta_{u 1} q_{, x} ; L_{4 p q}=\beta_{u 1} q_{, y} ; L_{7 p q}=\beta_{u 3} q_{, x} ; L_{8 p q}=\beta_{u 3} q_{, y} ; L_{9 p q}=\beta_{w 1} q$.

Equations 1, 2 of systems (16) and (17) describe the symmetric deformation of the plates, and $3,4,7-9,11$ - skew symmetry. Similarly, DE systems are obtained for other approximations.

The systems DE (15), (17) coincide for the corresponding plates., And (14) and (16) are structurally different only in the right parts. Therefore, each of the systems (14)-(17) can be divided into systems that separately describe the vortex boundary effect, the internal SSS, and the potential boundary effect. Methods of transformation and decoupling of such systems are given in [26].

In MSA, at each approximation, the general solutions must satisfy the same set boundary conditions. In the method of perturbations of geometric parameters [27] in a null approximation by a small parameter, the general solutions must satisfy the given boundary conditions, and in subsequent approximations the corresponding homogeneous boundary conditions.

4.3. Numerical results. The effectiveness of MSA was investigated in a boundary value problem for transversally isotropic shallow shells, freely fixed on the lateral surface (10). The transverse skew symmetric load $q(x, y)=q_{m n} \sin (m \pi x / a) \sin (n \pi y / b) \quad\left(q_{m n}-\right.$ const ) (DE (16) and (17) systems are considered). The following MGP were accepted: $G^{\prime} / G=0,1 ; E^{\prime} / E=1 ; a=b ; \quad v^{\prime}=v=0,3$; $m=n=1 ; k_{1}^{\prime} \neq 0 ; \quad k_{2}^{\prime} \neq 0 ; \quad R_{1}=R_{2} ; R_{1} / a=10 ; 20 ; 40 ; h / a=1 / 3 ; 1 / 5 ; 1 / 10$. Numerical results show that in the zero approximation of the difference between the SSS components and the results obtained by the direct solution of the DE equilibrium system, for $\sigma_{x}(x, y, z) / q(x, y)$ is less than $3.9 \%$, for $W(x, y, z) E /(q(x, y) h)-$ less than $1.1 \%$. In the first approximation for the difference does not exceed $1 \%$. This indicates a high convergence of MSA.

\section{Conclusions.}

1) The method of sequential approximations in MT of transversely isotropic shallow shells of arbitrary thickness is developed. In the zero approximation of MSA, the systems of equations for shells coincide with the equations for the corresponding plates. In the following approximations, the left parts are the same and coincide with the equations for the plates, and the right parts of the equations depend on the curvatures and components in the displacement components of the previous approximations.

2) By this method, the boundary value problem for the shell is reduced to a sequence of boundary value problems for the corresponding plates with symmetric and oblique deformation. Then inhomogeneous high-order DE can be reduced to low-order equations. MSA makes it easier to find a common solution for shallow shells.

3) Numerous studies have shown a high convergence of results.

4) MSA can be used to solve problems for shallow shells based on other theories. 


\section{REFERENCES}

1. Ambartsumyan S. A., 1974, The general theory of anisotropic shells, Moskva, Nauka

2. Burak Ja. J., Rudavsjkyj Ju. K., Sukhoroljsjkyj M. A., 2007, Analytical mechanics of locally loaded shells, Ljviv, Intelekt-Zakhid

3. Daouadj T. H., Adim B., 2017, Mechanical behaviour of FGM sandwich plates using a quasi-3D higher order shear and normal deformation theory, Structural Engineering and Mechanics, 61, 1, 49-63

4. Gulyaev V. I., Bazhenov V. A., Lizunov P. P., 1978, Non-classical theory of shells and its application to solving engineering problems, L'vov, L'vovskiy universitet

5. Jaiani G., 2015, Differential hierarchical models for elastic prismatic shells with microtemperatures, Journal of applied mathematics and mechanics, 95, 1, 77-90

6. Jemielita G., 1991, Plate Theory Meanders, Warsaw, Publisher of the Warsaw University of Teshnology

7. Kalekin O. Yu., 1965, To an approximate theory of shells of medium thickness, Prikladnaya mekhanika, 1, 12, 29-37

8. Kazemi M., 2018, Hygrothermoelastic buckling response of composite laminates by using mod fied shear deformation theory. Journal of Theoretical and Applied Mechanics, 56, 1, 3-14

9. Kil'chevskiy N. A., 1963, Basics of analytical shell mechanics, Kiev, AN USSR

10. Khoma I. Yu., 1986, The generalized theory of anisotropic shells, Kiev, Naukova dumka

11. Kulikov G. M., Plotnikova S. V., 2012, On the use of sampling surfaces method for solution of 3D elasticity problems for thick shells, Journal of applied mathematics and mechanics, 92, 11-12, 910-920

12. Kushnir R. M., Marchuk M. V., Osadchuk V. A., 2006, Nonlinear Problems of Static and Dynamics Susceptible to Transverse Shear and Compression of Plates and Shells, Aktual'nye problemy mekhaniki deformiruemogo tverdogo tela, Donetsk, Donetskiy universitet, 238-240

13. Libresku L. I., 1964, On the theory of anisotropic elastic shells and plates, Inzhenernyy zhurnal, 4, 3, 475-485

14. Nemish Yu. N., 2000, Development of analytical methods in three-dimensional problems of the statics of anisotropic bodies, Prykladna mekhanika, 36, 2, 3-38

15. Nemish Yu. N., Khoma I. Yu., 1991, Stress-strain state of non-thin shells and plates. Generalized theory, Prykladna mekhanika, 29, 11, 3-27

16. Plekhanov A. V., Prusakov A. P., 1976, On an asymptotic method for constructing a theory of bending of plates of medium thickness, Mekhanika tverdogo tela, 3, 84-90

17. Polizzotto C., 2018, A class of shear deformable isotropic elastic plates with parametrically variable warping shapes, Journal of applied mathematics and mechanics, 98, 2, 195-221

18. Ponyatovskiy V. V., To the theory of medium-thickness plates, Prikladnaya matematika i mekhanika, 24, 2, 335-341

19. Prusakov A. P., 1993, On the construction of twelfth-order bending equations for a transversely isotropic plate, Prikladnaya mekhanika, 29, 12, 51-58

20. Reissner E., 1944, On the theory of bending of elastic plates, Journal of Mathematics and Physics, 33, 184-191

21. Reissner E., 1950, On a variational theorem in elasticity, Journal of Mathematics and Physics, 33, 90-95

22. Reissner E., 1952, Stress strain relations in the theory of thin elastic shells. Journal of Mathematics and Physics, 31, 1, 32-42

23. Timoshenko S. P., 1921, On the correction for shear of the differential equation for transverse vibrations of prismatic bars, Philosophical Magazine and Journal of science, 41, 6, 245, 744-746

24. Vekua I. N., 1955, On a method for calculating prismatic shells, Trudy Tbilisskogo matematicheskogo instituta, 21, 191-293

25. Zelensjkyj A. Gh., 2007, The method of interconnected higher order equations in the analytical theory of shallow shells, Metody rozv'jazuvannja prykladnykh zadach mekhaniky deformivnogho tverdogho tila, Zbirn. nauk. pracj DNU, 8, 67-83

26. Zelensjkyj A. Gh., 2008, A method of solving a system of high-order differential equations in the analytical theory of non-thin shells, Metody rozv'jazuvannja prykladnykh zadach mekhaniky deformivnogho tverdogho tila, Zbirnyk naukovykh pracj Dnipropetrovsjkogho nacionaljnogho universytetu, 9, 93-103

27. Zelensky A. G., 2016, Method of Solution Equation System Within the Variant of Mathematical Theory of non-thin Shallow Shells, International Scientific Journal, Kiev, 7, 137-141

28. Zelensky A. G., 2019, Mathematical Theory of Transversally Isotropic Shells of Arbitrary Thickness at Static Load, Materials Science Forum, Actual problems of engineering mechanics, Trans Tech Publications Ltd, Switzerland, 968, 496-510 